\title{
Symbolic computation and perfect fluids in general relativity
}

\section{Sasha Cyganowski}

This thesis examines two areas in the field of general relativity.

The first area of discussion is that of relativistic perfect fluids. In particular, the following conjecture is considered:

Any shear-free, perfect fluid solution of Einstein's field equations in which the fluid satifies a barotropic equation of state $p=p(w)$ such that $w+p \neq 0$ is either nonexpanding or non-rotating.

The terms $p$ and $w$ above denote the pressure and total energy density of the perfect fluid, respectively.

Originally stated by Treciokas [10], the conjecture has been examined by many scientists, including Ellis [7], Collins [4], Carminati [1] and Senovilla, Sopuerta and Szekeres [9]. The conjecture has proved intractable to date, mainly due to the length and complexity of intermediate polynomials. In treating such polynomials, symbolic manipulators have proved to be of great assistance. In this thesis, a symbolic implementation of the Newman-Penrose formalism [8] is used to prove the conjecture in the following cases: Petrov type III spacetimes [2, 3], perfect fluids which obey the equation of state $p=-w / 3[6]$, and non-conformally flat spacetimes with the electric part of the Weyl tensor zero [6]. The conjecture is also examined for perfect fluids satisfying an equation of state $p=w / 9$.

Applications of the computer algebra system Maple to tetrad formalisms in general relativity comprise the second main area of discussion within this thesis. A Maple package is developed [5] which provides the following: functions which translate between the mathematically succint, Newman-Penrose formalism and the physically meaningful, orthonormal formalism; functions which perform null tetrad rotations; and a classification function which detemines the Petrov-Plebanski type of the Ricci tensor. Applications of the package, called NPTOOLS are also presented.

Received 22nd July, 1999

Thesis submitted to Deakin University, February 1999. Degree approved, June 1999. Supervisors: Dr. J. Carminati and Professor P. Kloeden.

Copyright Clearance Centre, Inc. Serial-fee code: 0004-9727/00 \$A2.00+0.00. 


\section{REFERENCES}

[1] J. Carminati, 'Shear-free perfect fluids in general relativity, I. Petrov type $N$ Weyl tensor', J. Math. Phys. 28 (1987), 1848-1853.

[2] J. Carminati and S.O. Cyganowski, 'Shear-free perfect fluids in general relativity: III. Petrov type III spacetimes', Classical Quantum Gravity 13 (1996), 1805-1817.

[3] J. Carminati and S.O. Cyganowski, 'Shear-free perfect fludis in general relativity: IV. Petrov type III spacetimes', Classical Quantum Gravity 14 (1997), 1167-1181.

[4] C.B. Collins, 'Shear-free perfect luids with zero magnetic Weyl tensor', J. Math. Phys. 25 (1984), 995-1000.

[5] S.O. Cyganowski and J. Carminati, 'The Maple package NPTOOLS; a symbolic algebra package for tetrad formalisms in general relativity', Comput. Phys. Comm. 115 (1998), 200-214.

[6] S.O. Cyganowski and J. Carminati, 'Shear-free perfect fludis in general relativity: Gravito-magnetic spacetimes', Gen. Relativity Gravitation (to appear).

[7] G.F.R. Ellis, 'Dynamics of pressure-free matter in general relativity', J. Math. Phys. 8 (1967), 1171-1194.

[8] E.T. Newman and R. Penrose, 'An approach to gravitational radiation by a method of spin coefficients', J. Math. Phys. 3 (1962), 566-578.

[9] J.M.M. Senovilla, C.F. Sopuerta and P. Szekeres, 'Theorems on shear-free perfect fludis with their Newtonian analogues', Gen. Relativity Gravitation 30 (1998), 389.

[10] R. Treciokas, Relativistic kinetic theory, Ph.D. Thesis (Cambridge University, 1972).

School of Computing and Mathematics

Deakin University

Warun Ponds Vic 3217

Australia

e-mail: sash@deakin.edu.au 